\title{
One-third Octave Bandpass Filter Algorithm of Overall Frequency-weighted Root Mean Square for Comfort Index Applied to Acceleration Sensor
}

\author{
Yan Jin, ${ }^{1,2,3}$ Liao Jian-bin, ${ }^{1,2,3}$ and Yu Hong-liang ${ }^{1,2,3 *}$ \\ ${ }^{1}$ School of Marine Engineering, Jimei University, \\ 176 Shigu Rd., Jimei District, Xiamen 361021, China \\ ${ }^{2}$ Fujian Engineering Research Center of Marine Engine Detecting and Remanufacturing, \\ 176 Shigu Rd., Jimei District, Xiamen 361021, China \\ ${ }^{3}$ Provincial Key Laboratory of Naval Architecture \& Ocean Engineering, \\ 176 Shigu Rd., Jimei District, Xiamen 361021, China
}

(Received May 31, 2019; accepted September 25, 2019)

Keywords: acceleration, overall frequency-weighted RMS, one-third band octave

Concerning the evaluation of vibration with regard to ship habitability, for overall frequencyweighted root mean square (RMS) acceleration, the frequency-weighted function is deduced, which is expressed as the product of three factors (high-pass filter, low-pass filter, and pure weighting function), and the filter is designed based on this function. According to the frequency weighting curve from the standard, a low-order continuous-time filter is designed by the optimal least-square method. The one-third octave filter is proposed and the designed progress is given. The method is applied to ship test acceleration data, because accelerometers are the most frequently used transducers to measure the vibration responses of structures, and then the overall frequency-weighted RMS acceleration is achieved, which agrees well with the standard filter and low-order continuous-time filter results.

\section{Introduction}

Shipboard vibration may interfere with duties and reduces the comfort of crew and passengers. The difference in comfort comes from people's different feelings about different vibration sources. ${ }^{(1)}$ To evaluate the habitability of different areas on a ship, the standard (ISO 6954) gives assessment guidelines for the evaluation of ship vibration with respect to habitability. ${ }^{(2)}$ The dynamic behavior of a structure can be used to verify its habitability, which can be related to the overall frequency-weighted root mean square (RMS) vibration, which can be easily and conveniently monitored by accelerometers. ${ }^{(3,4)}$

The calculation of the RMS of full frequency weighting is the most important task in the process of ship habitability vibration evaluation. Standards (ISO 6954 and ISO 2631) give the vibration acceleration frequency-weighted transfer function, ${ }^{(5,6)}$ which is expressed as a product of band limit, acceleration velocity transition, and upward step. Hayhoe transformed the transfer functions in standard (ISO 2631) into corresponding differential equations,

*Corresponding author: e-mail: yu1202@hotmail.com https://doi.org/10.18494/SAM.2019.2508 
established an evaluation computer program, and finally applied the method to Boeing 727-100 aircraft simulation. ${ }^{(6,7)}$ Zuo and Nayfeh proposed low-order continuous-time filters for the approximation of weightings, ${ }^{(8)}$ instead of high-order s-plane equations in standard (ISO 2631), ${ }^{(6)}$ and the low-order filter was used in the optimization of vehicle suspension. For the distortion signal using Zuo's IIR filter, ${ }^{(9)}$ Feng designed an FIR frequency weighting filter, which proved to be effective. ${ }^{(10)}$

In this work, we deduce the frequency-weighted transfer function in standards' form (ISO 2631) and fit the frequency weighting curve by Zuo's method. The one-third band octave filter is proposed and the designed progress is given, which is applied to ship test acceleration data, and then the overall frequency-weighted RMS acceleration is achieved. The results are compared with Zuo's and standard's methods (ISO 2631).

\section{ISO 8041 Method}

For translating vibration, habitability is evaluated using frequency weights, which is expressed as

$$
a_{w}=\left[\frac{1}{T} \int_{0}^{T} a_{w}^{2}(t) d t\right]^{\frac{1}{2}}
$$

Here, $a_{w}(t)$ is the weighted acceleration and $T$ is the measurement duration. Standard (ISO 2631) supplies the frequency weight for RMS calculation..$^{(2,5)}$ Table 1 gives the relationship of frequency weights with mid-frequencies, which only includes the $1-80 \mathrm{~Hz}$ band limit.

Table 1

Values of the frequency weighting with acceleration as the quantity.

\begin{tabular}{cccc}
\hline \multicolumn{2}{c}{ Frequency/Hz } & \multicolumn{2}{c}{ Acceleration as input quantity } \\
\hline Nominal & True & Factor & $\mathrm{dB}$ \\
\hline 1.00 & 1.000 & 0.833 & -1.59 \\
1.25 & 1.259 & 0.907 & -0.85 \\
1.60 & 1.585 & 0.934 & -0.59 \\
2.00 & 1.995 & 0.932 & -0.61 \\
2.50 & 2.512 & 0.910 & -0.82 \\
3.15 & 3.162 & 0.872 & -1.19 \\
4.00 & 3.981 & 0.818 & -1.74 \\
5.00 & 5.012 & 0.750 & -2.50 \\
6.30 & 6.310 & 0.669 & -3.49 \\
8.00 & 7.943 & 0.582 & -4.70 \\
10.00 & 10.000 & 0.494 & -6.12 \\
12.50 & 12.590 & 0.411 & -7.71 \\
16.00 & 15.850 & 0.337 & -9.44 \\
20.00 & 19.950 & 0.274 & -11.25 \\
25.00 & 25.120 & 0.220 & -13.14 \\
31.50 & 31.620 & 0.176 & -15.09 \\
40.00 & 39.810 & 0.140 & -17.10 \\
50.00 & 50.120 & 0.109 & -19.23 \\
63.00 & 63.100 & 0.0834 & -21.58 \\
80.00 & 79.430 & 0.0604 & -24.38 \\
\hline
\end{tabular}


Table 2

Factors and transfer functions of frequency weighting $\mathrm{Wm}$.

\begin{tabular}{|c|c|c|c|c|c|c|c|c|c|c|c|c|}
\hline Weights & \multicolumn{4}{|c|}{ Band limiting } & \multicolumn{3}{|c|}{$\mathrm{A}-\mathrm{V}$ transition } & \multicolumn{4}{|c|}{ Upward step } & Gain \\
\hline- & $\mathrm{f} 1 / \mathrm{Hz}$ & $\mathrm{Q}_{1}$ & $\mathrm{f} 2 / \mathrm{Hz}$ & $\mathrm{Q}_{2}$ & $\mathrm{f} 3 / \mathrm{Hz}$ & $\mathrm{f} 4 / \mathrm{Hz}$ & $\mathrm{Q}_{4}$ & $\mathrm{f} 5 / \mathrm{Hz}$ & $\mathrm{Q}_{5}$ & $\mathrm{f} 6 / \mathrm{Hz}$ & $\mathrm{Q}_{6}$ & - \\
\hline$W_{m}$ & $10-0.1$ & $\frac{1}{\sqrt{2}}$ & 100 & $\frac{1}{\sqrt{2}}$ & $\frac{1}{0.028 \times 2 \pi}$ & $\frac{1}{0.028 \times 2 \pi}$ & 0.5 & $\infty$ & 1 & $\infty$ & 1 & 1 \\
\hline
\end{tabular}

As shown in Table 2, the factors and transfer functions of the frequency weighting $W_{m}$ are given, which include band limit, $\mathrm{A}-\mathrm{V}$ transition, and upward-step filters.

\subsection{Band limit filter}

The band limit filter uses a combination of high-pass and low-pass second-order Butterworth filters, the components of which are defined as follows:

(a) high-pass transfer function

$$
H_{h}(s)=\frac{1}{1+\frac{\omega_{1}}{Q_{1} s}+\left(\frac{\omega_{1}}{s}\right)^{2}},
$$

(b) low-pass transfer function

$$
H_{l}(s)=\frac{1}{1+\frac{s}{Q_{2} \omega_{2}}+\left(\frac{s}{\omega_{2}}\right)^{2}} .
$$

Here, the $H_{h}(s)$ and $H_{l}(s)$ product is the band limit transfer function.

\section{$2.2 \quad$ A-V transition}

The $\mathrm{A}-\mathrm{V}$ transition filter is proportional to acceleration at lower frequencies and to velocity at higher frequencies. The expression is

$$
H_{t}(s)=\frac{\left(1+\frac{s}{\omega_{3}}\right) K}{1+\frac{s}{Q_{4} \omega_{4}}+\left(\frac{s}{\omega_{4}}\right)^{2}} .
$$

\subsection{Upward step filter}

The upward step filter has a steepness of $6 \mathrm{~dB}$ per octave, proportional to the acceleration, and its expression is 


$$
H_{s}(s)=\frac{1+\frac{s}{Q_{5} \omega_{5}}+\left(\frac{s}{\omega_{5}}\right)^{2}}{1+\frac{s}{Q_{6} \omega_{6}}+\left(\frac{s}{\omega_{6}}\right)^{2}}\left(\frac{\omega_{5}}{\omega_{6}}\right)^{2}
$$

\subsection{Overall frequency weighting filter}

The overall filter function of the weighted $W_{m}$ is the product of the band limit, accelerationvelocity transformation, and high-order filter, namely,

$$
H(s)=H_{h}(s) \times H_{l}(s) \times H_{t}(s) \times H_{s}(s) .
$$

$\omega_{1}, \ldots, \omega_{6}$ (where $\omega_{i}=2 \pi f_{i}, f_{i}$ are $f_{1}, \ldots, f_{6}$ in Table 1) represent angular frequencies, and $Q_{1}, Q_{2}$, $Q_{4}, Q_{5}$, and $Q_{6}$ are quality factors. $s$ (where $s=j 2 \pi f$ ) is a function of the imaginary angular frequency. The overall weighting $H(s)$ is determined by these parameters.

\subsection{Filter design}

We design the total frequency weighting function according to Eq. (6). As shown in Eq. (7), the function of the filter is plotted at each center frequency, as shown in Fig. 1. Compared with the $W_{m}$ curve provided by the standard, the agreement is good.

$H(s)=\frac{1.4151 \times 10^{7} \times s^{5}+8.8938 \times 10^{16} \times s^{4}+5.5882 \times 10^{26} \times s^{3}+1.9958 \times 10^{28} \times s^{2}-5.7184 \times 10^{28} \times s^{+}+2.6440 \times 10^{21}}{s^{8}+6.2832 \times 10^{9} \times s^{7}+3.9479 \times 10^{19} \times s^{6}+3.8247 \times 10^{22} \times s^{5}+1.8477 \times 10^{25} \times s^{4}+1.2919 \times 10^{27} \times s^{3}+2.8616 \times 10^{28} \times s^{2}+1.6982 \times 10^{29} \times s+4.9735 \times 10^{29}}$

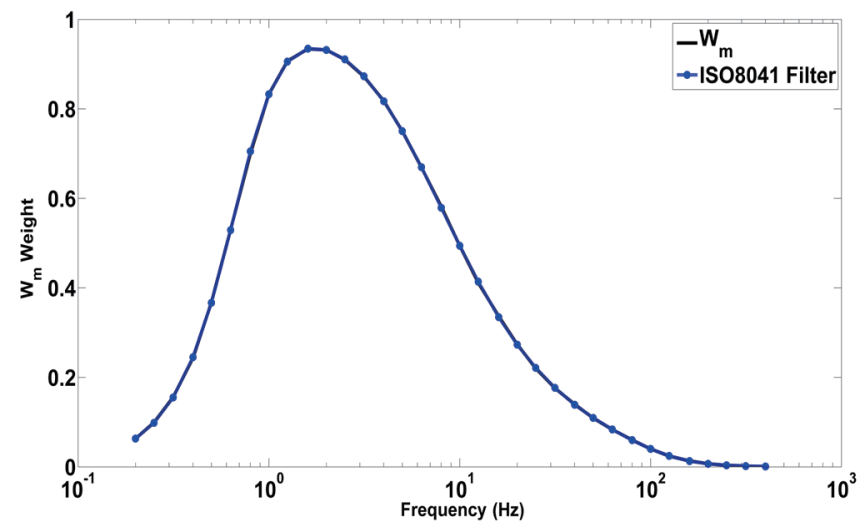

Fig. 1. (Color online) Frequency weighting $W_{m}$ curve compared with ISO8041 filter function. 


\section{Low-order Continuous-time Filter}

To design a low-order continuous-time filter, the magnitude $W_{i}$ of the frequency-weighting curve, which is a function of mid-frequency in the form of one-third octave, needs to be calculated, ${ }^{(2)}$ and its expression is

$$
W(\omega)_{\mid \omega=2 \pi f_{i}}=W_{i} \quad(i=1,2,3, \ldots, N) .
$$

The form of its transfer function is

$$
W(s)=\frac{b_{m} s^{m}+\cdots b_{1} s+b_{0}}{s^{n}+\cdots a_{1} s+a_{0}} .
$$

According to the frequency weighting curve from the standard, ${ }^{(2)}$ once the coefficients $\left(a_{0}\right.$, $\left.b_{0}, a_{1}, b_{1}, \ldots, b_{m}\right)$ of the transfer function are determined, the filter design can be completed.

The method adopted by Zuo is the least- $p$ th frequency domain fitting method, ${ }^{(8)}$ where $p$ is 2 for the least-square fitting and $p$ is gigantic for the Chebyshev fitting, and Eq. (10) needs to be minimized.

$$
\sum_{i=1}^{N}|| W\left(s_{i}\right)\left|-W_{i}\right|^{p}
$$

Here, $s_{i}=2 \pi j f_{i}$, mapping continuous time frequencies $\omega_{i}$ to $z$ domain by bilinear transformation, as in Eq. (11).

$$
\Omega_{i}=\frac{2}{T} \arctan \left(\frac{T \omega_{i}}{2}\right)=\frac{2}{T} \arctan \left(T \pi f_{i}\right)
$$

Here, $\Omega_{i}$ is the digital time frequency and $T$ is a constant and is chosen as 0.5 in the calculation process. The least-pth digital filter $W_{D}(z)$ is designed to minimize Eq. (10) and its expression is

$$
W_{D}(z)=\frac{\bar{b}_{n} z^{-n}+\cdots \bar{b}_{1} z^{-1}+\bar{b}_{0}}{z^{-n}+\cdots \bar{a}_{1} z^{-1}+\bar{a}_{0}} .
$$

The S-domain filter is obtained by bilinear transformation, as shown in Eq. (13).

$$
W(s)=\left.W_{D}(z)\right|_{z=(1+T s / 2) /(1-T s / 2)}
$$

The 4th- and 5th-order filters for approximating $W_{m}$ are shown in Eqs. (14) and (15). 


$$
\begin{gathered}
W^{(4)}(s)=\frac{35.62 s^{3}+545 s^{2}+471.37 s+227.12}{s^{4}+57.20 s^{3}+889.24 s^{2}+4859.73 s+12473.64} \\
W^{(5)}(s)=\frac{3.69 s^{5}+35.97 s^{4}+427.33 s^{3}+718.95 s^{2}+927.86 s+35.21}{s^{5}+54.41 s^{4}+744.93 s^{3}+4342.10 s^{2}+11619.69 s+11776.29}
\end{gathered}
$$

The fitting curves are compared with the $\mathrm{W}_{\mathrm{m}}$ curve, as shown in Fig. 2.

\section{One-third Octave Filter}

The exact mid-band frequency is $f_{c}$ for the one-third octave filter. The lower band-edge frequency $f_{l}$ and the upper band-edge frequency $f_{u}$ are determined from ${ }^{(11)}$

$$
\begin{gathered}
f_{l}=2^{-\frac{1}{6}} f_{c}, \\
f_{u}=2^{\frac{1}{6}} f_{c} .
\end{gathered}
$$

For a bandpass filter, the ratio of the filter bandwidth to the exact mid-band frequency is the normalized reference bandwidth $B_{r}{ }^{(12)}$ which is expressed as

$$
B_{r}=\left(f_{u}-f_{l}\right) / f_{c} .
$$

Substituting Eqs. (16) and (17) into Eq. (18), we obtain

$$
B_{r}=\left(2^{\frac{1}{6}}-2^{-\frac{1}{6}}\right)
$$

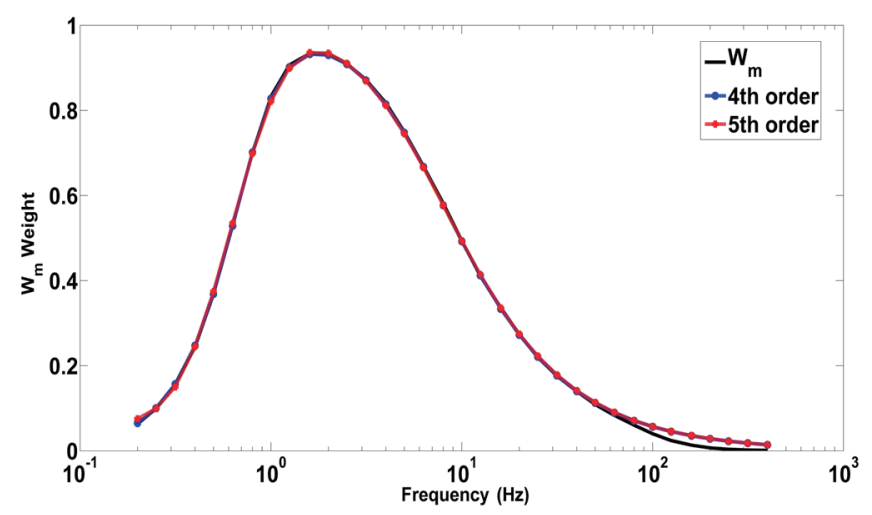

Fig. 2. (Color online) Frequency weighting $W_{m}$ curve with low-order continuous-time filters. 
For a one-third octave filter, the reference bandwidth quotient $\mathrm{Q}_{r}$ can be determined from

$$
Q_{r}=f_{c} / B_{r}=1 /\left(2^{\frac{1}{6}}-2^{-\frac{1}{6}}\right) .
$$

The design bandwidth quotient is larger than that for Butterworth filters, which is given by Eq. (21), where $n$ is the order of the filter.

$$
Q_{d}=[(\pi / 2 n) / \sin (\pi / 2 n)] Q_{r}
$$

Set the ratio of the upper and lower band-edge frequencies to the mid-band frequency as $\beta$, and it can be expressed by $Q_{d}$ as

$$
\beta=\left(1+\sqrt{1+\left(2 Q_{d}\right)^{2}}\right) / 2 Q_{d}
$$

The normalized cutoff frequencies are shown as follows:

$$
\begin{aligned}
& W_{l}=f_{c} /\left(f_{s} / 2\right) \beta, \\
& W_{u}=f_{c} \beta /\left(f_{s} / 2\right) .
\end{aligned}
$$

This is called the 'butter' function in MATLAB, in the form of

$$
[b, a]=\operatorname{butter}\left(n,\left(w_{l}, w_{u}\right)\right) .
$$

Here, $W_{l}$ and $W_{u}$ are from Eqs. (23) and (24), and $b$ and $a$ are the numerator and denominator coefficients of the filter, respectively. This is called the 'filter' function in MATLAB, as in the equation

$$
y_{i}=\operatorname{filter}(b, a, y) .
$$

Here, $y$ is the acceleration raw signal data and the result $y_{i}$ is filtered by applying a one-third octave filter. The overall frequency-weighted RMS acceleration is obtained by the summation of the squares of the weighted RMS spectral component $y_{i}$.

$$
a_{w}=\left[\sum_{i}\left(w_{i} y_{i}\right)^{2}\right]^{\frac{1}{2}}
$$

Here, $w_{i}$ is the weighting factor at the $i^{\text {th }}$ frequency band. 


\section{Ship Test Results}

We conducted a real ship test on a certain type of ship, and the measurement conditions are as follows: free-route test on a straight course, constant representative engine output, sea state 3 or less, full immersion of the propeller, and water depth not less than five times the draught of the ship.

The three-axis acceleration sensor is placed in the middle of the bridge floor to measure the vibration in three directions. Here, only the vibration acceleration results in the vertical direction are analyzed. The raw acceleration signal is filtered by the method recommended by ISO 8041, the one-third octave method, and the low-order filtering method separately. The filtered results are shown in Figs. 3-5. FFT is performed on the three results and comparisons are shown in Fig. 6.

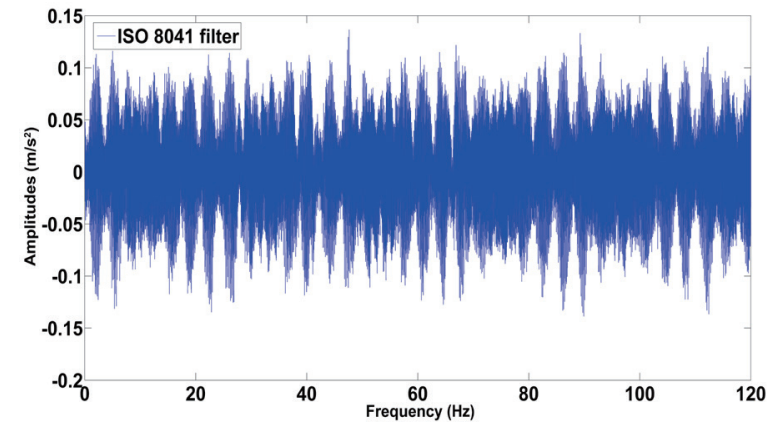

Fig. 3. (Color online) Weighted acceleration signal obtained using ISO 8041 filter.

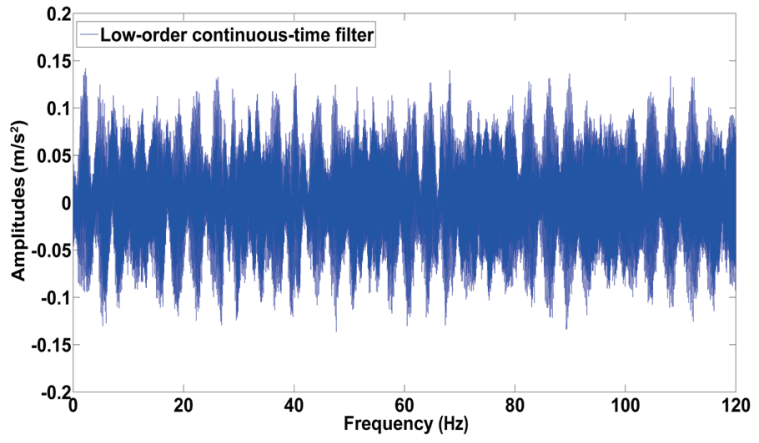

Fig. 4. (Color online) Weighted acceleration signal obtained using low-order continuous-time filter.

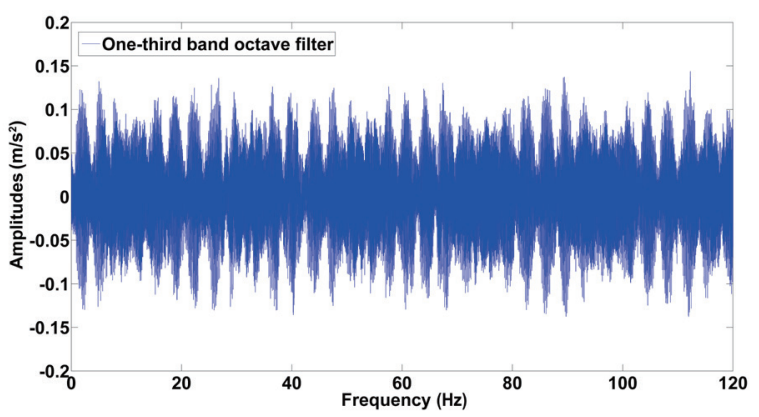

Fig. 5. (Color online) Weighted acceleration signal obtained using one-third band octave filter. 


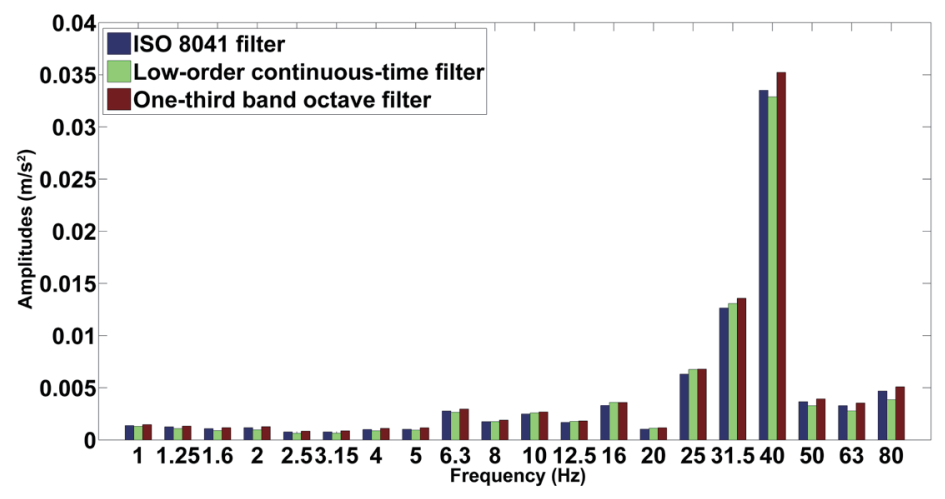

Fig. 6. (Color online) Comparison of one-third band octave.

Table 3

Comparison of overall frequency-weighted RMS accelerations.

\begin{tabular}{lcc}
\hline & Overall frequency-weighted RMS acceleration & Error \\
\hline ISO 8041 filter & $29.2367 \mathrm{~mm} / \mathrm{s}^{2}$ & - \\
Low-order continuous-time filter & $28.8723 \mathrm{~mm} / \mathrm{s}^{2}$ & $1.2464 \%$ \\
One-third band octave filter & $30.4017 \mathrm{~mm} / \mathrm{s}^{2}$ & $3.9847 \%$ \\
\hline
\end{tabular}

The comparison of the RMS calculation results of the full-frequency weighted acceleration of the three methods is shown in Table 3. The low-order continuous-time filter error is $1.2464 \%$ and the one-third octave filtering result is $3.9847 \%$, which is larger than the low-order error, but also agrees well. The overall frequency-weighted RMS acceleration is $30.4017 \mathrm{~mm} / \mathrm{s}^{2}$; according to the standard, ${ }^{(2)}$ this value indicates a conclusive view of the relationship between vibration magnitude and habitability. Many factors combine to determine the degree to which discomfort may be tolerated. An accurate assessment of the acceptability of the vibration and the formulation of vibration limits can only be made with the knowledge of many factors. Comfort expectations and annoyance tolerance are different in transportation vehicles compared with residential buildings. With regard to Table 3, passengers do not feel uncomfortable, and the shipboard vibration environment is commonly accepted.

\section{Conclusion}

To evaluate the overall frequency weighted RMS on a ship, on the basis of ship test acceleration signals, the derivation of the acceleration frequency weighting function in standard form is given firstly, and also the filter is designed for signal processing. The weights calculated using the filter are compared with those provided by the standard, which are in good agreement. The 4th- and 5th-order continuous-time analog filters are designed by the optimal least-pth frequency domain fitting method, which agrees well with standard weighted curves. A one-third octave filtering method is proposed, the method is applied to filter the acceleration time signal in the real ship test, and finally, the acceleration full-frequency weighted RMS is obtained. Compared with the previous two methods, the agreement is good, which can meet engineering needs. 


\section{Acknowledgments}

This work was supported by Fujian science and technology projects (No. 2017H0027).

\section{References}

1 Y. Q. Yang: J. Civ. Archit. Environ. Eng. 18 (2012) s2. http://kns.cnki.net/kcms/detail/detail. aspx?filename $=$ JIAN2012S2012 \&dbcode $=$ CJFQ \&dbname $=$ CJFD2012\&v $=$

2 ISO 6954: British Standards Document, BS ISO 20816-1, Mechanical vibration. Measurement and evaluation of machine vibration. General guidelines (2000). https://doi.org/10.3403/30328957u

3 S. Han: J. Mech. Sci. Technol. 24 (2010) 6. https://doi.org/10.1016/s0022-460x(88)80027-0

4 J. G. T Ribeiro, J. T. P. De Castro, and J. L. F Freire: Proc. 21st IMAC Conference and Exposition 2003 (IMAC, 2003). http://citeseerx.ist.psu.edu/viewdoc/download?doi=10.1.1.453.4304\&rep=rep1\&type=pdf

5 British Standards Document, BS ISO 2631-1, Mechanical vibration and shock. Evaluation of human exposure to whole-body vibration. General Requirements (2003). https://doi.org/10.3403/30197820u

6 BS EN ISO 8041:2005, Human response to vibration. Measuring instrumentation (2005). https://doi. org/10.3403/03281162

7 G. F. Hayhoe: Proc. 2014 FAA World-wide Airport Technology Transfer Conference (FAA, 2014). https://pdfs. semanticscholar.org/a50c/07765f873883a166d2ac424065731ea706b6.pdf

8 L. Zuo and S. A. Nayfeh: J. Sound. Vib. 265 (2003) 2. https://doi.org/10.1016/s0022-460x(02)01567-5

9 L. Zuo and P. S. Zhang: J. Vib. Acoust. 135 (2013) 1. https://doi.org/10.1115/1.4007562

10 D. Feng: Chin. J. Mech. Eng. 29 (2016) 4. https://doi.org/10.3901/cjme.2016.0321.034

11 ANSI S1.11: British Standards Document, BS 2475, Specification for octave and one-third octave band-pass filters (2004). https://doi.org/10.3403/00120907u

12 GBT 3241: BS EN 61260:1996, Electroacoustics. Octave-band and fractional-octave-band filters (2010). https:// doi.org/10.3403/02631503

\section{About the Authors}

Yan Jin received his $\mathrm{PhD}$ degree in marine engineering from Dalian Maritime University, Dalian, China, in 2013. He is now an assistant professor at Jimei University. His current research interests include modal analysis and vibration detection.

Liao Jian-bin is an assistant professor at Jimei University and a $\mathrm{PhD}$ candidate of Dalian Maritime University. He received his B.S. and M.S. degrees both in marine engineering from Jimei University in 1998 and 2007, respectively. His current research interests include marine engineering and fault diagnosis.

Yu Hong-liang received his PhD degree from Kobe University of Mercantile Marine, Kobe, Japan, in 2002. He is now a professor at Jimei University. His current research interests include naval architecture \& ocean engineering and marine engine detecting and remanufacturing. 